\title{
Análise da Prevalência de Entidades Coloproctológicas nos Pacientes Idosos do Serviço de Coloproctologia de um Hospital Universitário
}

\section{Analysis of the Prevalence of Coloproctological Conditions Elderly Patients of the Colorectal Unit in a University Hospital}

\author{
JULIANAFERREIRAMARTINS -FSBCP'; JULIANAGONÇALVES ROCHA-ASBCP'; ERON FÁBIO MIRANDA- \\ ASBCP $^{1}$; MARIACRISTINA SARTOR - TSBCP ${ }^{1}$; JULIANA STRADIOTTO STECKERT - FSBCP' ${ }^{2}$ ÁLVARO \\ STRADIOTTO STECKERT ${ }^{3}$; PAULO RICARDO BITTENCOURT GUIMARÃES ${ }^{4}$; PAULO GUSTAVO KOTZE-TSBCP ${ }^{5}$
}

${ }^{1}$ Médicos do Staff do SeCoHUC - Serviço de Coloproctologia do Hospital Universitário Cajuru - PUC-PR; ${ }^{2}$ Médica-residente do SeCoHUC; ${ }^{3}$ Acadêmico de medicina - Estagiário do SeCoHUC; ${ }^{4}$ Professor de Estatística da UFPR; ${ }^{5}$ Chefe do SeCoHUC.

MARTINS JF; ROCHA JG; MIRANDA EF; SARTOR MC; STECKERT JS; STECKERT AS; GUIMARÃES PRB; KOTZE PG. Análise da Prevalência de Entidades Coloproctológicas nos Pacientes Idosos do Serviço de Coloproctologia de um Hospital Universitário. Rev bras Coloproct, 2009;29(2): 145-157.

RESUMO: Introdução: projeções mostram que em 2020, os idosos constituirão cerca de $13 \%$ da população brasileira. Esta transformação não é apenas demográfica, mas também epidemiológica. Há real necessidade de maior compreensão da prevalência de determinadas doenças na faixa etária geriátrica. Objetivo: avaliar queixas e diagnósticos em coloproctologia mais prevalentes na população acima de 60 anos, comparando-os com os de idade inferior. Avaliar o número de idosos encaminhados a procedimentos cirúrgicos, suas comorbidades e analisar as complicações cirúrgicas, comparando-as ao grupo controle. Método: revisão de prontuários do ambulatório de Coloproctologia do Hospital Universitário Cajuru. Pacientes foram divididos em dois grupos: maiores de 60 anos de idade (idosos - grupo I) e menores de 60 anos (controles - grupo II). Os achados foram comparados entre os grupos. Resultados: foram incluídos 1126 pacientes, 19,36\% com mais de 60 anos. O número médio de queixas no grupo I foi de 1,21. As queixas mais frequentes nos idosos, com significância estatística foram: dor abdominal, constipação, diarreia e sangue oculto positivo. As doenças mais frequentes no grupo I foram: doença diverticular dos cólons, pólipos colônicos e câncer colorretal. No grupo I 58,36\% apresentavam alguma comorbidade. Não houve diferença significativa entre os grupos em relação às indicações cirúrgicas ou em relação às complicações pós-operatórias. Conclusões: os pacientes idosos apresentaram maior número médio de doenças diagnosticadas do que o grupo controle. Apresentaram também maior número de queixas colônicas e comorbidades associadas. Não houve diferença entre os dois grupos em relação às indicações cirúrgicas e às complicações pós-operatórias.

Descritores: Envelhecimento. Idoso. Cirurgia colorretal. Procedimentos cirúrgicos.

\section{INTRODUÇÃO}

O envelhecimento da população não é assunto novo. Países europeus, da América do norte e da Ásia (como China e Japão) já enfrentam esta realidade há longo período. O crescimento da população de idosos é um fenômeno mundial, e vem ocorrendo em um nível sem precedentes. Em 1950 existiam cerca de 204 mi-

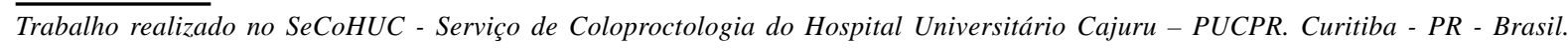


lhões de idosos no mundo. Cinco décadas depois, em 1998, este número havia aumentado para 579 milhões de pessoas ${ }^{1}$. As projeções da literatura especializada mostram que em 2050, esta população será de 1.9 bilhões de pessoas (o mesmo que a população infantil, de 0 a 14 anos) $)^{1}$. Atualmente, uma em cada dez pessoas tem 60 anos ou mais. Para 2050, estima-se que esta relação será de um para cinco em todo o mundo e de um para três nos países desenvolvidos ${ }^{1}$. De fato, o crescimento da população geriátrica é um fenômeno mais notado nos países em desenvolvimento, embora ainda não se tenha atingido as mesmas proporções já encontradas nos países desenvolvidos. Na América latina, o Brasil ocupa uma posição intermediária neste crescimento, com os idosos correspondendo a cerca de $8,6 \%$ da população ${ }^{1}$.

É difícil caracterizar uma pessoa como idosa tendo-se como critério único a idade. Este é um fator apenas cronológico, que não considera os fenômenos de natureza biológica, psíquica e social. No entanto, é um critério prático. No ocidente é comum associar-se o envelhecimento à saída da vida produtiva para a aposentadoria. Entretanto, em 1982, a Organização Mundial das Nações Unidas promoveu a primeira assembléia mundial sobre envelhecimento. Realizada em Viena, ela estabeleceu a idade de 60 anos como limite inferior para a velhice nos países em desenvolvimento e a idade de 65 anos nos países desenvolvidos ${ }^{3}$, norma igualmente recomendada pela Organização Mundial de Saúde.

Existe também subdivisão da população idosa, adotada por alguns autores, em dois grupos: os idosos (entre 55 e 75 anos) e os muito idosos (acima de 75 anos $)^{4}$. Este foi o segmento populacional que mais cresceu no último período intercensitário no Brasil, com crescimento de $49,3 \%$. Isto mostrou uma heterogeneidade de características dentro do grupo populacional idoso ${ }^{1}$.

Além das transformações demográficas, o Brasil tem experimentado uma transformação epidemiológica, com alterações relevantes no quadro da morbi-mortalidade ${ }^{6}$. Estas mudanças apontam para uma nova forma de "olhar" e de "cuidar" da população, em especial da população idosa. Embora este grupo etário tenha a expectativa de desfrutar de vários anos de suas vidas de forma saudável e ativa, é amplamente conhecido que eles são os maiores usuários dos serviços de saúde, principalmente aqueles vinculados ao Sistema Único de Saúde (SUS) ${ }^{5,6}$.

Os idosos apresentam mais problemas de saúde do que a população em geral $^{7}$. Em 1999, 73,2\% das pessoas que declararam ter consultado um médico nos últimos 12 meses tinham mais de 65 anos $^{7}$. Este foi também o grupo de maior coeficiente de internação hospitalar (14,8 por 100 pessoas do grupo $)^{7}$. Mais da metade dos idosos apresentam algum problema de saúde $(53,3 \%)$, sendo $23,1 \%$ portadores de doenças crônicas ${ }^{7}$. Grande parte dos pacientes gerontes busca atendimento médico em decorrência de suas doenças crônicas, mas cada vez mais pacientes idosos são encaminhados para avaliação de tratamento cirúrgico, nas mais variadas especialidades ${ }^{8}$. Na coloproctologia, muitas entidades clínicas têm sua frequência aumentada na população geriátrica. Grande parte dessas decorre do processo normal do envelhecimento, como a doença diverticular dos cólons e a incontinência fecal.

O objetivo principal deste estudo foi analisar as queixas e diagnósticos em coloproctologia mais prevalentes na população acima de 60 anos, atendida no ambulatório de Coloproctologia do Hospital Universitário Cajuru, comparando-se esta prevalência com os de idade inferior (grupo controle). Pretendeu-se também avaliar o número de pacientes idosos encaminhados a procedimentos cirúrgicos coloproctológicos, suas co-morbidades, e analisar as principais complicações cirúrgicas encontradas neste grupo de pacientes, comparando-as às do grupo-controle.

\section{MÉTODO}

Este estudo foi realizado no ambulatório do Serviço de Coloproctologia do Hospital Universitário Cajuru. Foram revisados todos os prontuários médicos de pacientes atendidos no referido ambulatório, entre janeiro de 2007 e maio de 2008.

O projeto de pesquisa foi aprovado pelo Comitê de Ética em Pesquisa da PUCPR (CEP - PUCPR), que permitiu a análise retrospectiva das informações do banco de dados composto pelos prontuários destes pacientes.

O ambulatório do Serviço de Coloproctologia do Hospital Universitário Cajuru, (SeCoHUC) foi criado em janeiro de 2007, data de início do programa de residência médica em coloproctologia desta instituição. Funciona no centro médico do Hospital Cajuru, com atendimentos 3 vezes por semana. Os pacientes são oriundos, em sua maioria, de encaminhamentos das Unidades Básicas de Saúde pela Secretaria Municipal de Saúde. São atendidos também pacientes encaminhados de outras cidades do estado do Paraná, por encaminhamentos da Secretaria Estadual de Saúde. Além disso, pacientes encaminhados por outras espe- 
Análise da Prevalência de Entidades Coloproctológicas nos Pacientes Idosos do Serviço de Coloproctologia de um Hospital Universitário Juliana Ferreira Martins e Cols.
Vol. 29 cialidades em atividade no Hospital Cajuru, como a Cirurgia Geral, Clínica Médica e Geriatria, são igualmente referidos e atendidos.

Durante o período citado, foram abertos 1310 prontuários. Destes, 184 foram excluídos: 22 por encaminhamento errôneo (as consultas foram agendadas para a especialidade errada pela Secretaria de Saúde); 44 por não terem retornado com os exames solicitados (e assim ainda não tinham diagnóstico confirmado); 14 prontuários não foram encontrados; 15 eram duplicados (dois prontuários para o mesmo paciente) e 89 prontuários foram abertos, mas os pacientes não compareceram à consulta.

Dos 1126 pacientes selecionados para inclusão no estudo, foram coletados dados de seus prontuários segundo protocolo em anexo. Foram registrados o nome, idade, sexo, queixas principais, diagnósticos principais e secundários, comorbidades, indicações cirúrgicas e complicações pós-operatórias. Estes pacientes foram então, divididos em dois grupos: os maiores de 60 anos de idade (idosos - grupo de estudo) e os menores de 60 anos. Os resultados encontrados na análise foram comparados entre os dois grupos.

Todos os pacientes atendidos no ambulatório de coloproctologia foram submetidos a anamnese geral e dirigida para a queixa principal, seguido de exame geral e proctológico (inspeção das regiões anal, perianal e perineal e retossigmoidoscopia rígida). Quando necessário foram solicitados exames complementares, como enema opaco, colonoscopia, ultra-sonografia e endoscopia digestiva alta. Pelo caráter da Instituição em que se realizou o estudo, universitária e de ensino, houve participação dos médicos residentes nas consultas e nos procedimentos cirúrgicos, sempre sob supervisão de um membro efetivo do SeCoHUC.

Para avaliação dos dados recorreu-se à analise descritiva com tabelas de frequência. Foram calculadas medidas-resumo como média, mediana e desvio padrão. Para testar as hipóteses dos objetivos do estudo foi utilizado o teste qui-quadrado, para comparação da frequência dos dois grupos de pacientes. Este teste foi empregado sempre que o número de casos foi suficiente. Para comparação entre média dos dois grupos foi empregado o teste $t$ de student para amostras independentes.

O nível de significância empregado (p) foi de 5\%.

\section{RESULTADOS}

Dos 1126 pacientes selecionados para o estudo, 218 tinham mais de 60 anos, totalizando 19,36\% do total (grupo I - idosos). A idade mínima nesse grupo foi de 61 anos e, a máxima, de 92 (média de 69,38 anos com desvio padrão de 6,65 anos).

Nesse grupo, $116(53,21 \%)$ eram do sexo feminino e 102 (46,79\%) do sexo masculino. Não houve diferença estatística significativa entre os sexos $(p=0,3452)$. Subdividindo-se a idade do grupo, conforme a literatura, entre idosos (até 75 anos) e "muito idosos" (com mais de 75 anos), verificou-se que aqueles constituem 83,94\% (183/218) do grupo, e estes $16,06 \%(35 / 218)$.

O outro grupo, constituído pelos menores de 60 anos (grupo II - controle), perfez um total de 908 indivíduos (80,64\% do total). A idade mínima foi de 1 ano e a máxima de 59 (média de 39,48 anos e desvio padrão de 13,54 anos). Neste grupo, 56,39\% (512/908) eram do sexo masculino e $43,61 \%$ (512/908) do sexo feminino. Houve diferença estatística significante entre os $\operatorname{sexos}(p=0,001)$.

Comparando-se os dois grupos, não houve diferença estatisticamente significante em relação ao sexo $(p=0,3964)$ (tabela 1$)$.

Quanto às principais queixas relatadas pelos pacientes (motivos que os levaram à consulta), verificou-se que o grupo abaixo de 60 anos (grupo II) apresentou, em média, 1,34 queixas por indivíduo (desvio padrão de 0,52), enquanto os idosos (grupo I) apresentaram média de 1,21 queixas (desvio padrão de 0,44 queixas). Assim, o grupo II apresentou número maior de queixas, com diferença estatística ( $p=0,000656)$ (tabela 2).

As principais queixas referidas pelos pacientes dos Grupos I e II encontram-se na tabela 3.

Comparando-se os dois grupos, verificou-se que as queixas mais frequentes nos idosos foram, de modo geral, ligadas ao cólon: dor abdominal $(p=0,001)$;

Tabela 1 - Comparação entre os grupos I e II, de acordo com o sexo e idade.

\begin{tabular}{llrc}
\hline & Sexo & $\mathbf{n}$ & \% \\
\hline Grupo I & Feminino & 116 & 53,21 \\
& Masculino & 102 & 46,79 \\
Grupo II & Feminino & 396 & 43,61 \\
& Masculino & 512 & 56,39 \\
Total & & $\mathbf{1 1 2 6}$ & $\mathbf{1 0 0}$ \\
\hline
\end{tabular}

$p=0,3964$ 
constipação $(p=0,02)$; diarreia $(p=0,046)$ e sangue oculto positivo $(p=0,004)$. Por outro lado, as queixas orificiais foram mais frequentes no grupo controle: sangramento anal $(p=0,002)$; aumento de volume anal $(p=0,001)$; dor anal $(p<0,0001)$ e secreção purulenta perianal $(p=0,04)$. Esses resultados encontram-se sumarizados na tabela 3 e no gráfico 1.

Quanto ao diagnóstico clínico, os idosos apresentaram número médio maior do que os pacientes do grupo controle. A média de diagnósticos por paciente no grupo I foi de 1,70 (com desvio padrão de 0,72 ), enquanto que no grupo II foi de 1,47 (desvio padrão de 0,64 diagnósticos por paciente). $\mathrm{O}$ valor de significância $(p)$ encontrado foi de 0,000003 (tabela 4).

Os diagnósticos foram realizados após anamnese, exame físico, exame coloproctológico e exames complementares (especialmente a colonoscopia). Os diagnósticos mais frequentes nos dois

Tabela 2 - Número médio de queixas, de acordo com os grupos.

\begin{tabular}{lcrc}
\hline & Média & n & D.P. \\
\hline Grupo I & 1,21 & 218 & 0,44 \\
Grupo II & 1,34 & 908 & 0,52 \\
Total & $\mathbf{1 , 3 1}$ & $\mathbf{1 1 2 6}$ & $\mathbf{0 , 5 1}$ \\
\hline
\end{tabular}

$p=0,000656$ grupos encontram-se sumarizados na tabela 5 e no gráfico 2.

Comparando-se os dois grupos, verificou-se que as doenças mais frequentes nos idosos, em relação ao grupo controle, com diferença estatística relevante, foram: doença diverticular $(p<0,0001)$; pólipos intestinais $(p<0,0001)$; câncer colorretal $(p<0,0001)$. Constipação, diarreia, retocele, colites inespecíficas, prolapso retal e megacólon, foram diagnósticos mais prevalentes nos pacientes acima de 60 anos. No entanto, a análise estatística não mostrou significância $(p>0,05)$. As doenças orificiais foram mais diagnosticadas nos indivíduos do grupo controle. A doença hemorroidária, embora tenha sido o diagnóstico mais comum nos dois grupos, foi mais prevalente nos pacientes mais jovens $(p=0,03)$. Também foram mais prevalentes, nesse grupo, com significância estatística, a fissura anal $(p=0,0013)$ e a fístula perianal $(p=0,015)$. Plicomas anais, síndrome do intestino irritável, condilomatose perianal e doença inflamatória intestinal foram mais frequentes nos pacientes mais jovens. No entanto, a análise estatística não mostrou significância. Esta comparação também se encontra descrita na tabela 5 .

$\mathrm{Na}$ análise das comorbidades, 162 pacientes foram excluídos por não haver dados nos seus prontuários sobre a presença ou não de doenças associadas. Destes, 141 eram do grupo II (portanto 15,53\% dos

Tabela 3 - Principais queixas. Em ordem decrescente de apresentação no grupo I.

\begin{tabular}{lrrrrrc}
\hline & \multicolumn{2}{c}{ Grupo I } & \multicolumn{2}{c}{ Grupo II } & Total & $p$ \\
& n & \multicolumn{1}{c}{$\boldsymbol{\text { I }}$} & n & \% & & \\
\hline Sangramento anal & 54 & 24,77 & 321 & 35,35 & 375 & $0,002^{*}$ \\
Aumento de volume anal & 33 & 15,14 & 230 & 25,33 & 263 & $0,001^{*}$ \\
Dor abdominal & 30 & 13,76 & 48 & 5,29 & 78 & $<0,0001^{*}$ \\
Constipação intestinal & 29 & 13,3 & 75 & 13,30 & 104 & $0,02^{*}$ \\
Dor anal & 28 & 12,84 & 280 & 30,84 & 308 & $<0,0001^{*}$ \\
Diarreia & 19 & 8,72 & 47 & 5,18 & 66 & $0,046^{*}$ \\
Pós-operatório de cirurgia colorretal & 19 & 8,72 & 15 & 1,65 & 34 & 0,0001 \\
Sangue oculto positivo & 15 & 6,88 & 26 & 2,86 & 41 & $0,004^{*}$ \\
Prurido anal & 8 & 3,67 & 50 & 5,51 & 58 & 0,27 \\
Incontinência fecal & 6 & 2,75 & 26 & 2,86 & 32 & 0,93 \\
Melena & 4 & 1,83 & 1 & 0,11 & 5 & - \\
Secreção purulenta perianal & 4 & 1,83 & 46 & 5,07 & 50 & $0,04^{*}$ \\
Pós-operatório de cirurgia orificial & 3 & 1,38 & 18 & 1,98 & 21 & - \\
Outras queixas & 10 & 4,59 & 18 & 1,98 & 28 & 0,02 \\
\hline
\end{tabular}




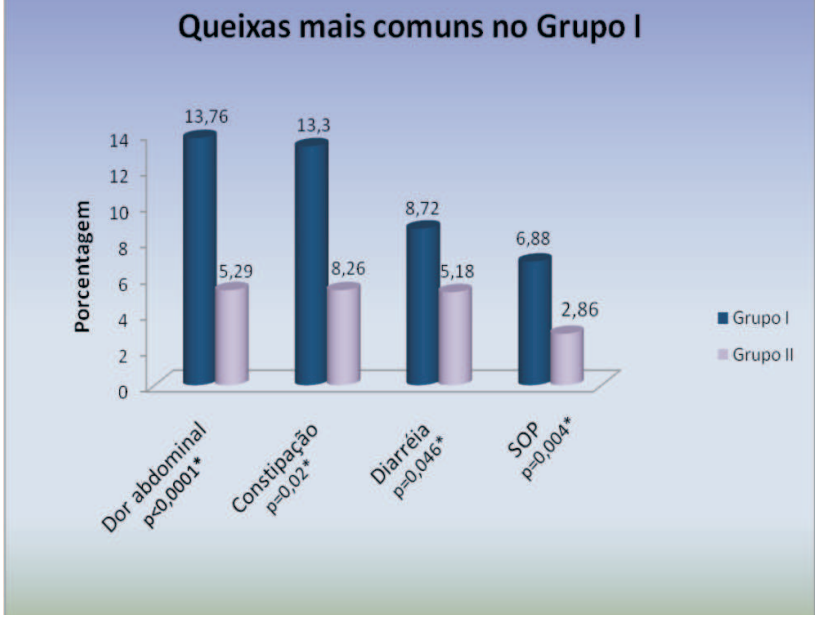

Gráfico 1 - Queixas mais comuns no Grupo I.

Tabela 4 - Número médio de diagnósticos de acordo com os grupos.

\begin{tabular}{lccc}
\hline & Média & n & D.P. \\
\hline Grupo I & 1,7 & 218 & 0,72 \\
Grupo II & 1,47 & 908 & 0,64 \\
Total & $\mathbf{1 , 5 1}$ & $\mathbf{1 1 2 6}$ & $\mathbf{0 , 6 6}$ \\
\hline
\end{tabular}

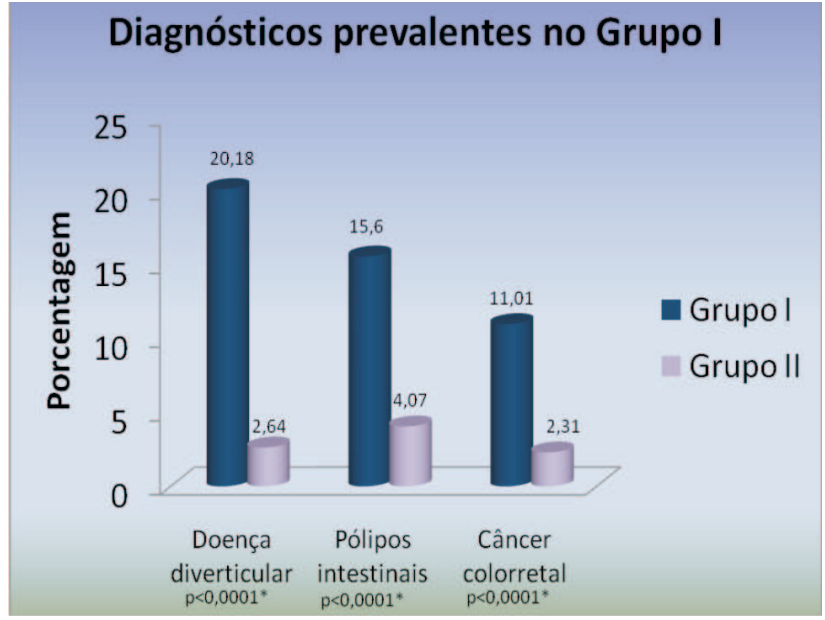

Gráfico 2 - Diagnósticos prevalentes no Grupo 1.

908 pacientes) e 21 eram do grupo I (9,63\% dos 218 pacientes).

Dessa maneira, para a análise desta variável, foram incluídos 964 pacientes. Formando o grupo geriátrico, obteve-se um total de 197 indivíduos. Desse montante, 58,36\% (115/197) apresentavam alguma comorbidade. Do grupo abaixo de 60 anos esta porcentagem foi menor, $31,68 \%$ (243/767) apresentavam

Tabela 5 - Principais diagnósticos. Em ordem decrescente de apresentação no grupo I.

\begin{tabular}{lrrrrrl}
\hline & \multicolumn{2}{c}{ Grupo I } & \multicolumn{2}{c}{ Grupo II } & Total & $p$ \\
& n & \multicolumn{1}{c}{$\%$} & n & \% & & \\
\hline Sangramento anal & 54 & 24,77 & 321 & 35,35 & 375 & $0,002^{*}$ \\
Hemorróidas & 91 & 41,74 & 454 & 50 & 545 & $0,03^{*}$ \\
Constipação Intestinal & 60 & 27,52 & 231 & 25,44 & 291 & 0,52 \\
Doença diverticular & 44 & 20,18 & 24 & 2,64 & 68 & $<0,0001^{*}$ \\
Pólipo & 34 & 15,6 & 37 & 4,07 & 71 & $<0,0001^{*}$ \\
Câncer de cólon & 24 & 11,01 & 21 & 2,31 & 45 & $<0,0001^{*}$ \\
Fissura anal & 14 & 6,42 & 151 & 16,63 & 165 & $0,0013^{*}$ \\
Plicoma & 12 & 5,5 & 83 & 9,14 & 95 & 0,08 \\
Diarreia & 9 & 4,13 & 25 & 2,76 & 34 & 0,29 \\
Retocele & 8 & 3,67 & 15 & 1,65 & 23 & - \\
Colite inespecífica & 6 & 2,75 & 6 & 0,66 & 12 & - \\
Incontinência fecal & 6 & 2,75 & 25 & 2,76 & 31 & 0,93 \\
SII & 6 & 2,75 & 41 & 4,52 & 47 & 0,242 \\
Fístula perianal & 4 & 1,83 & 53 & 5,84 & 57 & $0,015^{*}$ \\
Prolapso retal & 4 & 1,83 & 1 & 0,11 & 5 & - \\
Megacólon & 3 & 1,38 & 5 & 0,55 & 8 & - \\
DII & 2 & 0,92 & 14 & 1,54 & 16 & - \\
Outros & 39 & 17,89 & 111 & 12,22 & 150 & 0,027 \\
\hline
\end{tabular}

DII = Doença inflamatória intestinal; SII = Síndrome do Intestino Irritável . 
doenças associadas. Esta diferença teve significância estatística, $\operatorname{com} p<0,0001$.

A doença associada mais comum na população idosa foi a hipertensão arterial, acometendo 78,26\% (90/115). A hipertensão arterial foi também a comorbidade mais comum no grupo controle, e ocorreu em 53,91\% dos que tinham alguma doença associada (131/243). As demais doenças associadas encontradas nos dois grupos encontram-se na tabela 6 .

Comparando-se os dois grupos, verificou-se que a hipertensão arterial e a dislipidemia são significativamente mais frequentes nos idosos $(p<0,0001 \mathrm{e}$ $p=0,002$ respectivamente). Embora a diabetes mellitus, coronariopatia e arritmias cardíacas tenham sido mais frequentes em valor absoluto no grupo I, esta diferença não teve valor estatístico $(p>0,05)$. A depressão, por sua vez, foi um diagnóstico muito mais frequente do grupo mais jovem $(p=0,037)$. O hipotireoidismo, o refluxo gastro-esofageano e a epilepsia foram mais frequentes nos pacientes do grupo controle, mas esta diferença não teve significância estatística.

De todos os pacientes avaliados neste estudo, 237 tiveram indicação cirúrgica e efetivamente realizaram as operações indicadas $(21,05 \%$ dos 1126 pacientes). Destes, 40 pertenciam ao grupo I (18,35\% dos
218 idosos) e 197 eram do grupo controle $(21,70 \%$ dos 908 pacientes abaixo de 60 anos). Comparando os dois grupos em relação à indicação cirúrgica, verificamos que não há significância estatística $(p=0,19)$.

A principal operação realizada nos dois grupos foi a hemorroidectomia. As demais cirurgias realizadas estão sumarizadas na tabela 7.

Comparando-se os dois grupos, verificou-se que as operações mais realizadas nos idosos em relação ao grupo controle foram: retossigmoidectomia, fissurectomia, ligadura elástica de hemorroidas, colpoperineoplastia posterior, amputação abdominoperineal de reto. No entanto, não houve diferença estatisticamente significante em relação ao grupo controle $(p>0,05)$.

Dos 237 pacientes submetidos à cirurgia, 45 apresentaram algum tipo de complicação pós-operatória $(19,07 \%)$, alguns apresentaram mais de uma complicação. Destes, 10 eram idosos (25\% dos que foram operados) e 35 eram menores de 60 anos (17,35\% dos que foram operados). As complicações encontram-se resumidas na tabela 8 .

Apesar de não haver diferença estatística relevante entre os grupos $(p=0,26)$, o grupo I apresentou maiores índices de complicações, em número absoluto.

Tabela 6 - Comorbidades associadas. Em ordem decrescente de apresentação no grupo I.

\begin{tabular}{lrrrrrc}
\hline & \multicolumn{2}{c}{ Grupo I } & \multicolumn{2}{c}{ Grupo II } & Total & p \\
& n & \multicolumn{1}{c}{$\boldsymbol{\%}$} & \multicolumn{1}{c}{ n } & \% & & \\
\hline Sangramento anal & 54 & 24,77 & 321 & 35,35 & 375 & $0,002^{*}$ \\
HAS & 90 & 78,26 & 131 & 53,91 & 221 & $<0,0001^{*}$ \\
Diabetes mellitus & 21 & 18,26 & 34 & 13,99 & 55 & 0,29 \\
Dislipidemias & 16 & 13,91 & 11 & 4,55 & 27 & $0,002^{*}$ \\
AVC prévio & 10 & 8,7 & - & - & 10 & - \\
Depressão & 10 & 8,7 & 41 & 16,94 & 51 & $0,037^{*}$ \\
Coronariopatias & 8 & 6,96 & 10 & 4,12 & 18 & 0,25 \\
Hipotireoidismo & 6 & 5,22 & 18 & 7,41 & 24 & 0,44 \\
Arritmias & 6 & 5,22 & 10 & 4,12 & 16 & 0,64 \\
DRGE & 3 & 2,61 & 11 & 4,55 & 14 & - \\
DPOC & 3 & 2,61 & 11 & 4,55 & 14 & - \\
Epilepsia & 3 & 2,61 & 8 & 3,29 & 11 & - \\
Demência & 2 & 1,74 & - & - & 2 & - \\
Outras comorbidades & 22 & 19,13 & 63 & 26,03 & 85 & 0,15 \\
\hline
\end{tabular}

$H A S=$ Hipertensão arterial sistêmica $; A V C=$ Acidente vascular cerebral $;$ DRGE = Doença do refluxo gastroesofágico; DPOC = Doença pulmonar obstrutiva crônica. 
Tabela 7 - Procedimentos cirúrgicos realizados. Em ordem decrescente de frequência no grupo I.

\begin{tabular}{lcrrrr}
\hline & \multicolumn{2}{c}{ Grupo I } & \multicolumn{2}{c}{ Grupo II } & Total \\
& n & \% & n & \% & \\
\hline Hemorroidectomia & 14 & 35 & 88 & 44,67 & 102 \\
Retossigmoidectomia abdominal & 7 & 17,5 & 5 & 2,55 & 12 \\
Fissurectomia & 5 & 12,5 & 18 & 9,14 & 23 \\
Ligadura elástica & 4 & 10 & 18 & 9,14 & 28 \\
Fistulostomia & 3 & 7,5 & 30 & 15,23 & 33 \\
Colpoperineoplastia posterior & 2 & 5 & 4 & 2,03 & 6 \\
Retossigmoidectomia perineal & 2 & 5 & - & - & 2 \\
Amputação de reto & 1 & 2,5 & 2 & 1,02 & 3 \\
Ileostomia temporária & 1 & 2,5 & 2 & 1,02 & 3 \\
Esficteroplastia & 1 & 2,5 & 5 & 2,54 & 6 \\
Cauterização de condilomatose & 1 & 2,5 & 7 & 3,55 & 8 \\
Ressecção de cisto pilonidal & - & - & 7 & 3,55 & 7 \\
Ressecção de hidradenite supurativa & - & - & 6 & 3,05 & 6 \\
Colectomia direita & - & - & 4 & 2,03 & 4 \\
Reconstrução de trânsito & - & - & 3 & 1,53 & 3 \\
Drenagem de abscesso & - & - & 2 & 1,02 & 2 \\
Cirurgia de Hartmann & - & - & 1 & 0,51 & 1 \\
Outras cirurgias & 2 & 5 & 7 & 3,57 & 9 \\
\hline
\end{tabular}

$p=0,19$

Tabela 8 - Complicações cirúrgicas. Em ordem decrescente de frequência no grupo I.

\begin{tabular}{lrrrrr}
\hline & \multicolumn{2}{c}{ Grupo I } & \multicolumn{2}{c}{ Grupo II } & Total \\
& n & \% & n & \% & \\
\hline Sem complicações & 30 & 75 & 162 & 82,65 & 192 \\
Fissura residual & 1 & 10 & 20 & 58,82 & 21 \\
Seroma & 2 & 20 & 4 & 11,76 & 6 \\
Hérnia incisional & 1 & 10 & 3 & 8,57 & 4 \\
Estenose & 1 & 10 & 2 & 5,88 & 3 \\
Infecção de ferida operatória & - & - & 3 & 8,57 & 3 \\
Incontinência fecal & 1 & 10 & 2 & 5,88 & 3 \\
Fistuleta anal & - & - & 2 & 5,88 & 2 \\
Evisceração & 1 & 10 & 1 & 2,85 & 2 \\
Oclusão intestinal & 1 & 10 & 1 & 2,85 & 2 \\
Prolapso do estoma & 1 & 10 & - & - & 1 \\
Fístula entérica & 1 & 10 & - & - & 1 \\
Deiscência do estoma & - & - & 1 & 2,85 & 1 \\
\hline
\end{tabular}

$p=0,26$

\section{DISCUSSÃO}

O envelhecimento da população é realidade em todo o mundo, e o Brasil não foge a esta situação. Esse processo tem origem nas transformações sociais e econômicas vividas pelas nações desenvolvidas desde o século passado. Hoje, enfrenta-se os resultados destas modificações ${ }^{6}$. O cenário atual é o de grandes mudan- 
Análise da Prevalência de Entidades Coloproctológicas nos Pacientes Idosos do Serviço de Coloproctologia de um Hospital Universitário Juliana Ferreira Martins e Cols. ças, não só pela alteração demográfica já mencionada, mas também pelo desenvolvimento significativo da ciência e tecnologia. Este é sem dúvida, um dos grandes responsáveis pela maior sobrevida e maior expectativa de vida. Muitas doenças, há pouco tempo consideradas letais, principalmente para o idoso, são hoje bem controladas ou totalmente curadas. Estima-se que esta evolução técnica e científica permita ao ser humano alcançar os 110 ou 120 anos de idade ainda neste século $^{13}$.

No Brasil, segundo dados do IBGE, cerca de $8,6 \%$ da população tem mais de 60 anos de idade ${ }^{1}$. Ainda segundo a mesma fonte, a cidade de Curitiba possui cerca de $8,4 \%$ de sua população constituída por idosos. No presente estudo, a porcentagem de pacientes gerontes atendidos em um período consecutivo de 16 meses, foi de $19,36 \%$. Destes, $53,21 \%$ eram do sexo feminino. Embora não tenha havido diferença estatisticamente significativa entre os sexos $(p=0,3452)$, o maior número absoluto de mulheres atendidas pode ser reflexo de uma tendência mundial de feminização da população idosa. Este fenômeno pode ser explicado pela maior expectativa de vida das mulheres, que no Brasil é, em média, oito anos maior do que nos homens. De fato, no Brasil, para cada 100 mulheres idosas, há 81,6 homens idosos ${ }^{1}$.

Dentro da população geriátrica existe um segmento, conhecido por "muito idosos", que são aqueles indivíduos com mais de 75 anos. Esta é uma população especial entre os gerontes, pois foi a faixa que mais cresceu durante o último período intercensitário (aumento de 49,3\%). É tendência mundial. Nesta casuística o grupo correspondeu a $16,06 \%$ dos idosos. É um dado extremamente relevante, como bem observado por Souza Filho em $2000^{11}$, pois é um grupo com incidência mais elevada de doenças associadas e com maior frequência de emergências cirúrgicas. Tal fato, associado à menor capacidade funcional decorrente do processo normal do envelhecimento, torna o grupo especialmente vulnerável a qualquer agressão cirúrgica.

Neste trabalho, entre os pacientes abaixo de 60 anos, a maior parte $(56,39 \%)$ era do sexo masculino $(p=0,001)$. No entanto, quando comparou-se os dois grupos, não houve diferença estatística significativa em relação ao sexo $(p=0,3964)$. A idade média neste grupo foi de 39,48 anos (com desvio padrão de 13,54 anos). De fato, nesta idade, há ainda mais homens do que mulheres. Ao nascimento, a expectativa de vida das mulheres é muito superior à dos homens. Na adoles- cência os homens alcançam as mulheres e após este período a vantagem das mulheres em relação à expectativa de vida se torna mais pronunciada ${ }^{1}$.

Quanto às principais queixas relatadas pelos pacientes, verificou-se que o grupo geronte apresentou média de 1,34 por indivíduo (+/- 0,52), enquanto o grupo controle apresentou média de 1,21 queixas (+/$0,44)$. Assim, o grupo de pacientes mais jovens apresentou um número médio de queixas superior aos ido$\operatorname{sos}(p=0,000656)$. Este dado é conflitante quando analisamos os diagnósticos efetivamente realizados. Aqui, os idosos apresentaram número médio maior do que os pacientes do grupo controle. A média de diagnósticos realizados por paciente no grupo dos idosos foi de 1,70, enquanto que no grupo controle foi de 1,47 , com significância estatística.

As queixas referem-se ao motivo da consulta, relatadas pelo próprio paciente. Entretanto, os diagnósticos são realizados após anamnese, exame físico, exame coloproctológico e exames complementares, como a colonoscopia. É provável que o acanhamento, por parte do idoso, seja um dos motivos desta diferença. Lenardt et al. $(2007)^{16}$, observaram em seu trabalho, visível constrangimento dos idosos quando submetidos a procedimentos invasivos. Embora o exame proctológico (inspeção, toque retal e retossigmoidoscopia) faça parte do exame mínimo da especialidade, muitos pacientes idosos o temem. Assim, deixam de relatar alguns sintomas. Por outro lado, o maior número de diagnósticos em relação às queixas reforça a importância da anamnese detalhada, exame físico completo e a solicitação de exames complementares direcionados sempre que necessário. A valorização das queixas e a observância de protocolos de prevenção de câncer colorretal foi possivelmente um dos motivos do maior número de diagnósticos encontrados.

A principal queixa encontrada nos dois grupos foi o sangramento anal (em 24,77\% dos idosos e 35,35\% dos controles). Este é sintoma que assusta o paciente, pela temida associação com neoplasias, o que o leva a procurar auxílio médico precoce para esclarecimento do diagnóstico. Embora tenha sido a queixa mais comum nos dois grupos, foi mais significante nos mais jovens $(p=0,002)$.

Comparando-se os dois grupos, pôde-se observar que as queixas mais frequentes na população geriátrica estudada e que tiveram diferença estatística significativa, foram de origem colônica. Dor abdominal foi relatada por $13,76 \%$ dos gerontes contra $5,29 \%$ dos 
jovens ( $p=0,001)$. Constipação foi detectada em 13,30\% do grupo I contra $8,26 \%$ no grupo II $(p=0,02)$. Diarreia ocorreu em $8,72 \%$ dos gerontes contra $5,18 \%$ dos jovens $(p=0,046)$. Encaminhamentos por sangue oculto positivo corresponderam ao motivo de consulta de $6,88 \%$ dos maiores de 60 anos, enquanto que apenas 2,86 dos controles se apresentaram por esse motivo $(p=0,004)$.

As queixas mais comuns no grupo controle foram, de modo geral, de origem orificial, como: aumento de volume anal $(p=0,001)$; dor anal $(p<0,0001)$ e secreção purulenta perianal $(p=0,04)$. Camilleri et al., em sua revisão de literatura em 2000 , verificaram que estudos epidemiológicos sobre sintomas gastrointestinais em idosos, mostram uma alta prevalência de queixas compatíveis com distúrbios funcionais do trato gastrointestinal ${ }^{17}$.

No presente estudo, o diagnóstico clínico mais realizado foi de doença hemorroidária. Este foi o achado mais comum nos dois grupos $(41,74 \%$ nos idosos e $50 \%$ dos mais jovens), no entanto, foi estatisticamente mais significante nos pacientes do grupo controle $(p=0,03)$. Fissura anal, e fístula perianal também foram mais prevalentes neste grupo ( $p=0,0013$ e $p=0,015$ respectivamente).

Cruz et al., revisando 34.000 pacientes com queixas coloproctológicas em 2006, relatou achado de doença hemorroidária como diagnóstico principal em $27,3 \%$ dos $\operatorname{casos}^{18}$. Entretanto, relatos da literatura confirmam um declínio da incidência desta doença a partir dos $65 \operatorname{anos}^{19}$. Isto contraria a teoria do "deslizamento do assoalho anal" defendida por Haas et al. em $1984^{20}$ que justificaria o aumento das hemorroidas ao longo do envelhecimento. Pinho et al. em 2002, avaliaram prospectivamente 2323 pacientes atendidos em um ambulatório de coloproctologia. Estes autores encontraram $44 \%$ de diagnósticos anorretais (hemorroidas, trombose perianal, fissura, fístulas e plicomas). Assim como no presente estudo, notou-se que a frequência destas doenças declina com o avançar da idade e questionou-se a origem degenerativa e caráter evolutivo da doença hemorroidária ${ }^{21}$.

Em relação às doenças coloproctológicas mais prevalentes no idoso, em comparação com o grupo controle, verificou-se que as de origem colônica foram as mais relevantes. A doença diverticular foi diagnosticada em 20,18\% dos idosos, e em apenas $2,64 \%$ dos mais jovens $(p<0,0001)$. A incidência de diverticulose, nos países ocidentais de fato aumenta com a idade ${ }^{22}$. Estima-se que sua incidência seja de $5 \%$ nos pacientes com 40 anos, podendo aumentar para até cerca de $80 \%$ aos 80 anos $^{23}$.

Outro diagnóstico dos mais prevalentes no idoso foi o de pólipos intestinais. Em 15,60\% dos gerontes foram identificados pólipos contra $4,07 \%$ dos pacientes abaixo de 60 anos $(p<0,0001)$. Alguns estudos demonstraram que a incidência de pólipos intestinais aumenta com a idade, assim como a displasia destas lesões. No entanto o aumento da incidência após os 80 anos é controverso ${ }^{24}$. Em pacientes assintomáticos, estima-se que a incidência de pólipos seja de 24 a $47 \%$ aos $50 \operatorname{anos}^{25}$. A colonoscopia é o método de escolha para detecção dos pólipos e prevenção do câncer colorretal. Este exame é seguro nesta população com taxas de complicação de aproximadamente $0,6 \%$, e não deve ser deixado de ser realizado quando necessário ${ }^{26}$. Há, no entanto, controvérsias em relação à idade em que a prevenção deve cessar ${ }^{27}$.

O diagnóstico de câncer colorretal ocorreu em $11,01 \%$ dos idosos, enquanto no grupo abaixo de 60 anos em apenas $2,31 \%$. O valor de significância encontrado foi menor que 0,0001 . Há evidências de que o câncer colorretal no idoso está aumentando, que a abordagem desses pacientes está mudando para um tratamento mais agressivo e multimodal ${ }^{28}$. A maior faixa de incidência desta neoplasia encontra-se entre os 50 e 70 anos $^{29}$. Apesar de ser predominante no idoso, sua frequiência nos pacientes jovens é encontrada em 2 a $6 \%$ deste grupo $^{30}$. Os achados do presente estudo são equivalentes a estes dados.

Outras doenças, como constipação, diarreia, retocele, colites inespecíficas, prolapso retal e megacólon foram mais prevalentes nos pacientes idosos, mas a análise estatística não mostrou significância $(p>0,05)$. No entanto, é importante se observar que a constipação foi o segundo diagnóstico mais prevalente neste grupo $(27,52 \%)$. Esta é uma situação muito comum no idoso. Camilleri et al., em sua revisão de literatura, encontraram prevalência de $24,1 \%$ nos gerontes ${ }^{17}$. Estes números podem aumentar para cerca de $44 \%$ quando avaliamos apenas idosos interna$\operatorname{dos}^{31}$. No entanto, a constipação na população geriátrica parece ser multifatorial, e não decorrente apenas do processo de envelhecimento orgânico. Está relacionada com baixa ingesta de fibras, sedentarismo, uso de medicamentos constipantes, e uma variedade de doenças neurológicas que afetam a inervação do intesti$\mathrm{no}^{22}$. 
Apesar do processo de envelhecimento não estar, necessariamente, relacionado a doenças e incapacidades, as doenças crônicas são mais frequentemente encontradas entre os idosos ${ }^{32}$. Da população geriátrica avaliada neste trabalho, 58,36\% apresentavam alguma comorbidade, a média foi de 1,73 doenças associadas por paciente. No grupo controle $31,68 \%$ apresentavam alguma comorbidade.

Comparando-se os dois grupos, verificou-se que a hipertensão arterial e a dislipidemia são significativamente mais frequentes no idoso do que no grupo controle $(p<0,0001$ e $p=0,002$ respectivamente). A hipertensão foi encontrada em 78,26\% dos gerontes. Estudos de prevalência desta doença, geralmente são realizados em grupos específicos e são limitados. Lourenço et al. (2002) encontraram uma prevalência de 53,3\% de hipertensos nos usuários da Universidade Aberta da Terceira Idade no Rio de Janeiro ${ }^{33}$. Prevalência semelhante foi encontrada por Alves et al., em São Paulo, em trabalho publicado em $2007^{32}$.

A dislipidemia foi encontrada em $13,91 \%$ dos idosos, enquanto os mais jovens apresentaram esta comorbidade em apenas 4,55\%. O diabetes mellitus, coronariopatia, arritmias cardíacas e acidentes vasculares cerebrais prévios foram mais prevalentes nos idosos, no entanto a análise estatística não mostrou significância relevante entre os grupos.

A única comorbidade associada que foi mais prevalente no grupo abaixo de 60 anos, com significância estatística, foi a depressão $(p=0,037)$. O hipotireoidismo, o refluxo gastro-esofágico e a epilepsia foram igualmente mais frequentes nos menores de 60 anos. Entretanto, esta diferença não teve significância estatística. Embora as síndromes depressivas e demenciais sejam os problemas mentais mais prevalentes na população idosa ${ }^{7}$, a população geronte estudada não apresentou grande número destas entidades.

Muitos trabalhos na literatura apontam a cirurgia no idoso como factível e segura. A idade não parece ser isoladamente responsável pelo aumento de complicações. Entretanto, as comorbidades associadas e a diminuição da capacidade funcional, quando evidentes, podem alterar o desfecho cirúrgico ${ }^{34}$. Greenfield em 1975, já observara que os índices de mortalidade dos pacientes idosos estavam decrescendo significativamente quando comparados às décadas anteriores ${ }^{35}$.
Nesse estudo, o achado comparativo entre os dois grupos relacionados à indicação cirúrgica não mostrou diferença estatística. De todos os pacientes, $21,05 \%$ realizaram algum tipo de procedimento cirúrgico (237/1126). Do grupo geronte, 18,35 \% foram operados. Essa porcentagem no grupo controle foi de $21,70 \%(p=0,19)$. A principal indicação cirúrgica nos dois grupos foi a hemorroidectomia $(35 \%$ dos idosos que fizeram alguma cirurgia e em 44,67\% dos mais jovens que fizeram alguma cirurgia). Não houve, no entanto, diferença estatisticamente significativa entre os dois grupos $(p=0,26)$.

$\mathrm{O}$ fato de que o presente estudo compara um grupo com até 60 anos e outro com 60 anos ou mais pode não ter sido ideal para mostrar as diferenças entre jovens e idosos. Para isto, seria necessário comparar um grande número de pacientes. Esta dificuldade já foi relatada por diversos autores ${ }^{30}$.

Küster Filho et al. fizeram uma comparação da população geriátrica operada no Hospital de Clínicas de Curitiba em um intervalo de dez anos. Estes autores observaram que em 1989, num período de doze meses consecutivos, $9,7 \%$ de todos os pacientes operados tinham idade igual ou superior a 65 anos. Dez anos após, em 1999 essa porcentagem foi de 21,1\%. Um crescimento de $11,4 \%{ }^{8}$. Santos Junior (2003), relatou que dos últimos 376 pacientes operados por ele, $45 \%$ tinha idade superior a $61 \operatorname{anos}^{9}$. Bravo Neto et $a l$, verificaram que $19,65 \%$ dos pacientes operados no período de um ano, em um serviço de coloproctologia tinham mais de 60 anos $^{37}$.

A habilidade em minimizar, reconhecer e tratar complicações é um dos aspectos mais importantes da prática cirúrgica. A idade não é isoladamente fator de aumento da morbidade. No entanto, quando associadas à diminuição de reservas funcionais decorrentes de doenças associadas pode elevar o número de complicações.

No presente estudo, encontramos taxa de complicação pós-operatória de 19,07\%. Alguns pacientes apresentaram mais de uma complicação. Apesar de não haver diferença estatisticamente relevante entre os grupos $(p=0,26)$, os gerontes apresentaram mais complicações $(25 \%$ dos que foram operados contra $17,35 \%$ no grupo controle) em número absoluto.

Em revisão recente, Torres Neto et al. observaram taxa de complicação cirúrgica pós-operatória geral em $47,1 \%$ dos pacientes operados em Serviço de 
Análise da Prevalência de Entidades Coloproctológicas nos Pacientes Idosos do Serviço de Coloproctologia de um Hospital Universitário

Juliana Ferreira Martins e Cols.
Vol. 29 $\mathbf{N}^{\circ} 2$
Coloproctologia com residência médica na especialidade $^{38}$.

Bravo Neto et al. encontraram taxa de complicação em idosos de $21 \%$ quando as cirurgias foram realizadas de maneira eletiva, e de 63,63\% quando o caráter cirúrgico foi de urgência ${ }^{37}$. Küster Filho et al. encontraram morbidade de $9 \%$ em cirurgias eletivas e de $21 \%$ nas emergências, em análise de 391 pacientes idosos operados no Serviço de Cirurgia Geral do Hospital de Clinicas de Curitiba ${ }^{39}$. Os resultados encontrados no presente estudo corroboram os achados da literatura.

Todos os pacientes idosos avaliados neste trabalho foram submetidos a cirurgias eletivas. No entanto, é importante observar a necessidade de se reduzir ao máximo possível o índice de procedimentos cirúrgicos de emergência na população geriátrica. Como bem observado por Souza Filho (2000), “deixar de operar eletivamente o idoso aumentará o risco de morte por possível necessidade de tratamento de emergência" ${ }^{11}$.

\section{CONCLUSÕES}

Os idosos apresentam número maior de comorbidades em relação aos mais jovens na população estudada. Hipertensão arterial sistêmica e dislipidemia foram estatisticamente relevantes.

Houve predomínio de diagnósticos e queixas de origem colônica nos pacientes do grupo geronte. Os pacientes mais jovens apresentaram maior incidência de diagnósticos e queixas anorretais.

Não houve diferença estatística significativa em relação às indicações cirúrgicas e às complicações pósoperatórias entre os dois grupos.

\section{AGRADECIMENTOS}

Agradecemos aos Doutores Renato Araújo Bonardi (Chefe da Unidade de Coloproctologia e do Serviço de Cirurgia Geral do HC - UFPR) e José Mário Tupiná de Machado (Chefe do Serviço de Geriatria do HUC - PUCPR) pelas sugestões dadas a este trabalho.

\begin{abstract}
Introduction: It is expected that in 2020, elderly people will reach $13 \%$ of the Brazilian population. This involves epidemiologic and medical concerns. There is few data in the literature regarding colorectal and anal diseases in this population. Objective: to compare data about colorectal and anal conditions in elderly people (older than 60 years) with younger patients, including associated diseases, surgical procedures and complications. Method: The charts of the patients of the outpatient colorectal unit were retrospectively reviewed. They were divided in two groups: older than 60 years (group I) and younger than 60 years (controls, group II). Results: 1126 patients were included in this study. 19,36\% were older than 60 years. The average number of complaints in the group I was 1,21. Abdominal pain, constipation, diarrhea and positive fecal occult blood test were more frequent in the elderly. Anorectal complaints were more prevalent in the control group. The more prevalent conditions in group I, with statistical significance, were: diverticular disease, colorectal polyps and colorectal cancer. In group I, 58,36\% had associated diseases. There was no statistical significance between the groups regarding surgical procedures and complications. Conclusions: Colonic diseases were more prevalent in the elderly group. They had more associated diseases compared with younger patients. There was no statistical difference between the groups regarding surgical treatment and complications.
\end{abstract}

Key words: Ageing. Elderly. Colorectal surgery. Surgical procedures.

\section{REFERÊNCIAS}

1. IBGE. Perfil dos Idosos responsáveis pelos Domicílios no Brasil. Rio de Janeiro: Ministério de Planejamento, Orçamento e Gestão: Diretoria de Pesquisa: 2002.

2. Barbosa JMM, Dias RC, Pereira LSM. Qualidade de vida e estratégias de enfrentamento em idosos com incontinência fecal: uma revisão da literatura. Rev Bras Geriatr Gerontol 2007;10(3):ISSN1809-9823.

3. Organização das Nações Unidas, Assembléia Mundial sobre Envelhecimento: resolução 39/125. Viena ;1982.
4. Wissow LS, Belote A, Kramer W, Compton-Phillips A, Kritzler R, Weiner JP. Promoting advance directives among elderly primary care patients. J Gen Intern Med 2004;19:944-951.

5. Fiedler MM, Peres KG. Functional status and associated factors among the elderly in a southern brazilian city: a population-based study. Cad. Saúde Pública 2008;24(2):409415.

6. Veras RP. Novos paradigmas do modelo assistencial no setor de saúde: conseqüência da explosão populacional dos idosos no Brasil. In: Veras RP. Terceira idade: gestão contemporânea em saúde. Rio de Janeiro, Relume Dumará, 2002. p.11-79. 
7. Garrido R, Menezes PR. O Brasil está envelhecendo: boas e más notícias por uma perspectiva epidemiológica. Rev bras Psiquiatr 2002;24(1):3-6.

8. Küster-Filho ACC, Nóbrega NL, Souza Filho ZA. Estudo comparativo da população geriátrica cirúrgica no Hospital de Clinicas, Curitiba - UFPR, no intervalo de dez anos (19891999). Rev Bras med 2002;59(6):475-478.

9. Santos JR JMC. O paciente cirúrgico idoso. Rev Bras Coloproct 2003;23(4):305-316.

10. Hofer SM, Sliwinski MJ. Understanding ageing. Gerontology 2001;47:341-352.

11. Souza Filho ZA. Avaliação do risco cirúrgico em octogenários. 2000. 49 f. Dissertação (Professor Titular de Clínica Cirúrgica) - Pontifícia Universidade Católica do Paraná, Curitiba, 2000.

12. Kohn RR Cause of death in very old people. JAMA 1982;247(20):2793-2797.

13. Veras RP, Caldas CP. Promovendo a saúde e a cidadania do idoso: o movimento das universidades da terceira idade. Ciência \& Saude Coletiva 2004;9(2):423-432.

14. Kalache A. Envelhecimento populacional e as informações de saúde do PNAD: demandas e desafios contemporâneos. Posfácio. Cad Saude Pública 2007;23(10): 2503-2505.

15. Petroianu A, Pimenta LG. In: Petroianu A. Clinica e cirurgia geriátrica. Rio de Janeiro, Guanabara-Koogan 2002.

16. Lenardt $\mathrm{MH}$, Hammerschimidt KAS, Pívaro ABR, Borghi CS. Os idosos e os constrangimentos nos eventos da internação cirúrgica. Texto Contexto Enferm 2007;16(4):737-745.

17. Camilleri M, Lee JS, Viramontes B, Bharucha AE, Tangalos EG. Insights into the pathophysilogy and mechanisms of constipation, irritable bowel disease, and diverticulosis in older people. J Am Geriatr Soc 2000;48(9):1142-1150.

18. Cruz GMG, Santana JL, Santana SKA, Ferreira RMG, Neves PM, Faria MNZ. Doenças anais concomitantes à doença hemorroidária: revisão de 1222 pacientes. Rev bras Coloproct 2006;26(3):369-376.

19. Nivatvongs S. Hemorrhoids. In: Nivatvongs S, Gordon PH. Principles and Practice of Surgery for the Colon, Rectum and Anus. New York, Informa Healthcare, 2007. p.144-166.

20. Haas PA, Fox TA Jr, Haas GP. The pathogenesis of hemorrhoids. Dis colon rectum 1984;27(7):442-450.

21. Pinho MS, Ferreira LC, Vasconcelos ECG, Souza Filho NA, Reis MC. Análise da prevalência por sexo e idade nas doenças anorretais frequientes. Rev bras Coloproc 2002; 3:158163.

22. Shamburek RD, Farrat JT. Disorders of the digestive system in the elderly. N Engl J Med 1990;322(7):438-443.

23. Thorson AG, Goldberg SM. Benign colon: diverticular disease. In: The ASCRS Textbook of Colon and Rectal Surgery. New York, Springer Science 2007. p.269-285.

24. O’Brien MJ, Winamer SJ, Zauber AG, Bushey MT, Sternberg SS, Gottieb LS, et al. The National Polyp Study.
Patient and polpyp characteristcs associated with highgrade dysplasia in colorectal adenomas. Gastroenterology 1990;98:371-379.

25. Burnstein NJ, Hicks TC. Polyps. In: The ASCRS Textbook of Colon and Rectal Surgery. New York, Springer Science 2007. p.362-372.

26. Duncan JE, Sweeney WB, Trudel JL, Madoff RD, Mellgren AF. Colonoscopy in the elderly: low risk, low yield in asymptomatic patients. Dis Colon Rectum 2006;49(5):646651.

27. Holt PR. Gastrointestinal diseases in the elderly. Curr Opin Clin Nutr Metab Care 2003;6:41-48.

28. Araújo DV, Cirrincione A. custo do tratamento do câncer colorretal em pacientes idosos. Rev Bras Geriatr Gerontol 2006;9(1):ISSN1809-9823.

29. Instituto Nacional do Câncer (Brasil): Prevenção do câncer do intestino. Rev bras Cancerol 2003;49:207.

30. Malheiros APR, Teixeira MG, Habr-Gama A, Alcântara PSM. Resultados do tratamento cirúrgico do câncer colo-retal em doentes de idade até 64 anos e de 65 anos ou mais. Rev bras Coloproct 2005;25(2):128-136.

31. Gatusso JM, Kamm MA. Review article: the management of constipation in adults. Aliment Pharmacol Ther 1993;7:487500 .

32. Alves LC, Leimann BCQ, Vasconcelos MEL, Carvalho MS, Vasconcelos AGG, Fonseca TSO et al. A influência das doenças crônicas na capacidade funcional dos idosos do Município de São Paulo, Brasil. Cad Saúde Pública 2007;23(8):1924130.

33. Lourenço RA, Veras RP, Silva NAS. Hipertensão Arterial e outros fatores de risco de doenças cardiovascular: prevalências em uma população de idosos. In: Veras RP. Terceira idade: gestão contemporânea em saúde. Rio de Janeiro, Relume Dumará, 2002. p.97-161.

34. Vironem JH, Sanio P, Husa AI, Kellokumpu IH. Complications and Survival after surgery for rectal cancer in patients younger than and aged 75 years or older. Dis Colon Rectum 2002;47:1225-1231.

35. Greenfield IJ. Results os surgery in the aged. In: Greenfield IJ. Surgery in the aged. Philadelphia. Saunders,1975.p.139145 apud Saenger ME et al. Análise das internações cirúrgicas em idosos, em um hospital geral. Arq med 2005;8(2):519.

36. Gallagher P, Clark K. The ethics of surgery in the elderly demented patient with bowel obstruction. J Med Ethics 2002;28:105-108.

37. Bravo Neto GP, Bravo TP, Vieira OM. Infecção pós-operatória no paciente idoso. Ars crvandi 1986;19(9):83-93.

38. Torres Neto JR, Souza Junior MCA, Santiago RR, Prudente ACL. Cirurgias colorretais no Hospital Universitário da Universidade Federal de Sergipe: três anos de criação do Serviço de Coloporctologia (Série histórica). Rev bras Coloproct 2008;28(1):77-83. 
39. Küster-Filho ACC, Nóbrega NL, Souza Filho ZA. Estudo comparativo da população geriátrica cirúrgica no Hospital de Clinicas, Curitiba - UFPR, no intervalo de dez anos (19891999) apud Souza Filho ZA. Avaliação do risco cirúrgico em octogenários. 2000. 49 f. Dissertação (Professor Titular de Clínica Cirúrgica) - Pontifícia Universidade Católica do Paraná, Curitiba, 2000.
Endereço para correspondência: JULIANA FERREIRA MARTINS

Rua Alferes Poli, 2325 - casa A

Rebouças - Curitiba - PR

CEP 80220-050

Fone/Fax: (41) 3022-5500

E-mail: jufmartins@hotmail.com 